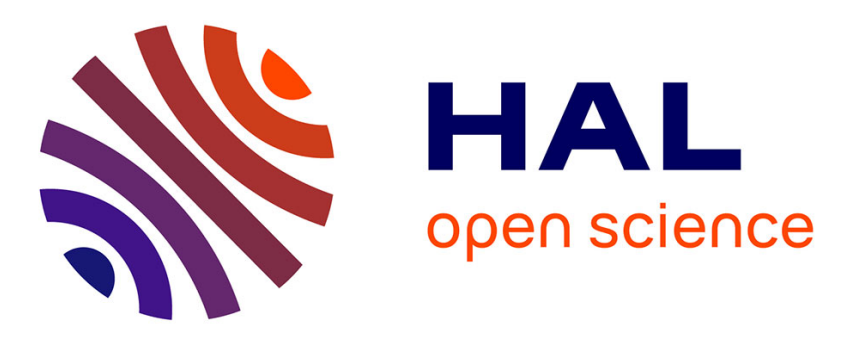

\title{
A uniformly stable finite difference space semi-discretization for the internal stabilization of the plate equation in a square
}

Karim Ramdani, Takeo Takahashi, Marius Tucsnak

\section{- To cite this version:}

Karim Ramdani, Takeo Takahashi, Marius Tucsnak. A uniformly stable finite difference space semidiscretization for the internal stabilization of the plate equation in a square. ENUMATH 2005 - The 6th European Conference on Numerical Mathematics and Advanced Applications, Jul 2005, Santiago de Compostela, Spain. pp.1068-1076, 10.1007/978-3-540-34288-5_107 . hal-00140479

\author{
HAL Id: hal-00140479 \\ https://hal.science/hal-00140479
}

Submitted on 26 Jan 2016

HAL is a multi-disciplinary open access archive for the deposit and dissemination of scientific research documents, whether they are published or not. The documents may come from teaching and research institutions in France or abroad, or from public or private research centers.
L'archive ouverte pluridisciplinaire HAL, est destinée au dépôt et à la diffusion de documents scientifiques de niveau recherche, publiés ou non, émanant des établissements d'enseignement et de recherche français ou étrangers, des laboratoires publics ou privés. 


\title{
A uniformly stable finite difference space semi-discretization for the internal stabilization of the plate equation in a square
}

\author{
Karim Ramdani ${ }^{1}$, Takéo Takahashi ${ }^{2}$, and Marius Tucsnak ${ }^{3}$ \\ 1 IECN (Université Henri Poincaré) and INRIA ramdani@loria.fr \\ ${ }^{2}$ IECN (Université Henri Poincaré) and INRIA takahash@iecn.u-nancy.fr \\ 3 IECN (Université Henri Poincaré) and INRIA tucsnak@iecn.u-nancy.fr
}

Summary. We propose a finite difference space semi-discretization of the stabilized Bernoulli-Euler plate equation in a square. The scheme studied yields a uniform exponential decay rate with respect to the mesh size.

\section{Statement of the main result}

Consider a square plate $\Omega=(0, \pi) \times(0, \pi)$ subject to a feedback force distributed on a rectangular subdomain $\mathcal{O}=[a, b] \times[c, d]$ of $\Omega$. If $\chi_{\mathcal{O}}$ denotes the characteristic function of $\mathcal{O}$, the stabilization problem considered reads:

$$
\begin{cases}\ddot{w}(t)+\Delta^{2} w(t)+\chi_{\mathcal{O}} \dot{w}(t)=0, & x \in \Omega, t>0, \\ w(t)=\Delta w(t)=0, & x \in \partial \Omega, t>0, \\ w(x, 0)=w_{0}(x), \quad \dot{w}(x, 0)=w_{1}(x), & x \in \Omega .\end{cases}
$$

It is well known (cf. [3]) that the energy $E(t)=\|\dot{w}(t)\|_{L^{2}(\Omega)}^{2}+\|\Delta w(t)\|_{L^{2}(\Omega)}^{2}$ of system (1) decreases exponentially. The aim of this paper is to propose a space semi-discretization of this internal stabilization problem that ensures an exponential decay of the discretized energy $E_{h}(t)$ which is uniform with respect to the mesh size. This is not a trivial issue because of the possible appearance during the approximation process of high frequency spurious modes that cannot be damped by the feedback term. The appearance of such spurious modes in the approximation by finite differences or finite elements of control problems has been emphasized in several works (see, for instance [1], [2], [6] and the review paper [7]). Various solutions to overcome this difficulty have been proposed in the literature. The one followed in this paper is the one based on the introduction of an artificial numerical viscosity term.

Let us now precise the numerical scheme proposed. Given $N_{1} \in \mathbb{N}$, denote by $h=\pi /\left(N_{1}+1\right)$, and assume that there exist integers $a(h), b(h), c(h), d(h)$ in $\left\{1, \ldots, N_{1}\right\}$ such that 


$$
a=a(h) h, \quad b=b(h) h, \quad c=c(h) h, \quad d=d(h) h .
$$

Let $w_{j, k}$ denote for all $j, k \in\left\{0, N_{1}+1\right\}$ the approximation of the solution $w$ of system (1) at the point $x_{j, k}=(j h, k h)$. We use the standard finite difference approximation of the laplacian, by setting for all $j, k \in\left\{1, \ldots, N_{1}\right\}$ :

$$
\Delta w(j h, k h) \approx \frac{1}{h^{2}}\left(w_{j+1, k}+w_{j-1, k}+w_{j, k+1}+w_{j, k-1}-4 w_{j, k}\right) .
$$

Set $V_{h}=\mathbb{R}^{\left(N_{1}\right)^{2}}$ and let $w_{h} \in V_{h}$ be the vector whose components are the $w_{j, k}$ for $1 \leq j, k \leq N_{1}$. In order to satisfy the boundary conditions in (1), we impose that

$$
\forall k \in\left\{0, \ldots, N_{1}+1\right\}:\left\{\begin{array}{l}
w_{0, k}=w_{k, 0}=w_{N_{1}+1, k}=w_{k, N_{1}+1}=0 \\
w_{-1, k}=-w_{1, k}, w_{N_{1}+2, k}=-w_{N_{1}, k}, \\
w_{k,-1}=-w_{k, 1}, w_{k, N_{1}+2}=-w_{k, N_{1}} .
\end{array}\right.
$$

The matrix $A_{0 h}$ representing the discretization of the bilaplacian with hinged boundary conditions is defined via its square root $A_{0 h}^{\frac{1}{2}}$ given by

$$
\left(A_{0 h}^{\frac{1}{2}} w_{h}\right)_{j, k}=-\frac{1}{h^{2}}\left(w_{j+1, k}+w_{j-1, k}+w_{j, k+1}+w_{j, k-1}-4 w_{j, k}\right),
$$

for all $1 \leq j, k \leq N_{1}$. The finite-difference space semi-discretization for system (1) studied in this paper reads then

$$
\begin{cases}\ddot{w}_{j, k}+\left(A_{0 h} w_{h}\right)_{j, k}+\left(\chi_{\mathcal{O}} \dot{w}_{h}\right)_{j, k}+h^{2}\left(A_{0 h} \dot{w}_{h}\right)_{j, k}=0, & 1 \leq j, k \leq N_{1}, \\ w_{j, k}(0)=w_{0 h}, \quad \dot{w}_{j, k}(0)=w_{1 h}, & 1 \leq j, k \leq N_{1},\end{cases}
$$

In the above equations, $w_{0 h}$ and $w_{1 h}$ are suitable approximations of the initial data $w_{0}$ and $w_{1}$ on the finite-difference grid and $\chi_{\mathcal{O}} \dot{w}_{h}$ denotes the vector of $V_{h}$ whose components are the $\dot{w}_{j, k}$ if $j$ and $k$ are such that $x_{j, k} \in \mathcal{O}$, and 0 otherwise. The numerical viscosity term $h^{2} A_{0 h} \dot{w}_{h}$ in (4) is introduced in order to damp the high frequency modes. Our main result is the following.

Theorem 1. The family of systems defined by (3)-(4) is uniformly exponentially stable, i.e. there exist constants $C, \alpha, h^{*}>0$ (independent of $h, w_{0 h}$ and $w_{1 h}$ ) such that :

$$
\left\|\dot{w}_{h}(t)\right\|^{2}+\left\|A_{0 h}^{\frac{1}{2}} w_{h}(t)\right\|^{2} \leq C e^{-\alpha t}\left(\left\|w_{1 h}\right\|^{2}+\left\|A_{0 h}^{\frac{1}{2}} w_{0 h}\right\|^{2}\right)
$$

for all $h \in\left(0, h^{*}\right)$ and all $t>0$.

In the above theorem and in the remaining part of this paper, we denote by $\|\cdot\|$ the Euclidean norm in $\mathbb{R}^{m}$ for various values of $m$. The proof of theorem 1 is based on the following frequency domain characterization for the uniform exponential stability of a sequence of semigroups (see [4, p.162]). 
Theorem 2. Let $\left(\mathbb{T}_{h}\right)_{h>0}$ be a family of semigroups of contractions on the Hilbert space $V_{h}$ and $A_{h}$ be the corresponding infinitesimal generators. The family $\left(\mathbb{T}_{h}\right)_{h>0}$ is uniformly exponentially stable if and only if the two following conditions are satisfied:

i) For all $h>0, i \mathbb{R} \subset \rho\left(A_{h}\right)$, where $\rho\left(A_{h}\right)$ denotes the resolvent set of $A_{h}$, ii) $\sup _{h>0, \omega \in \mathbb{R}}\left\|\left(i \omega-A_{h}\right)^{-1}\right\|<+\infty$.

\section{Proof of Theorem 1}

\subsection{Abstract second and first order formulations}

Let $U_{h}=\mathbb{R}^{(b(h)-a(h)+1) \times(d(h)-c(h)+1)}$ be the discretized input space, where the integers $a(h), b(h), c(h)$ and $d(h)$ are defined by $(2)$. If $B_{0} \in \mathcal{L}\left(L^{2}(\mathcal{O}), L^{2}(\Omega)\right)$ denotes the restriction operator defined by $B_{0} u=\chi_{\mathcal{O}} u$ for all $u \in L^{2}(\mathcal{O})$, we introduce its finite-difference approximation $B_{0 h} \in \mathcal{L}\left(U_{h}, V_{h}\right)$ by setting for all $u_{h} \in U_{h}:\left(B_{0 h} u_{h}\right)_{j, k}=u_{j, k}$ if $j$ and $k$ are such that $x_{j, k} \in \mathcal{O}$, and 0 otherwise. The adjoint $B_{0 h}^{*} \in \mathcal{L}\left(V_{h}, U_{h}\right)$ of $B_{0 h}$ is then defined for all $w_{h} \in V_{h}$ by $\left(B_{0 h}^{*} w_{h}\right)_{j, k}=w_{j, k}$ for all $j, k$ such that $x_{j, k} \in \mathcal{O}$.

The finite-difference semi-discretization (3)-(4) admits the following abstract second order formulation:

$$
\left\{\begin{array}{l}
\ddot{w}_{j, k}+\left(A_{0 h} w_{h}\right)_{j, k}+\left(B_{0 h} B_{0 h}^{*} \dot{w}_{h}\right)_{j, k}+h^{2}\left(A_{0 h} \dot{w}_{h}\right)_{j, k}=0, \quad 1 \leq j, k \leq N_{1}, \\
w_{j, k}=\left(A_{0 h}^{\frac{1}{2}} w_{h}\right)_{j, k}=0, \quad j, k=0, N_{1}+1 \\
w_{j, k}(0)=w_{0 h}, \quad \dot{w}_{j, k}(0)=w_{1 h}, \quad 0 \leq j, k \leq N_{1}+1 .
\end{array}\right.
$$

It can be easily checked that the sequence $\left(\left\|B_{0 h}\right\|_{\mathcal{L}\left(U_{h}, V_{h}\right)}\right)$ is bounded and that the eigenvalues of $A_{0 h}^{\frac{1}{2}}$ are

$$
\lambda_{p, q, h}=\frac{4}{h^{2}}\left[\sin ^{2}\left(\frac{p h}{2}\right)+\sin ^{2}\left(\frac{q h}{2}\right)\right], \text { for } 1 \leq p, q \leq N_{1} .
$$

A corresponding sequence of normalized eigenvectors is given by the vectors $\varphi_{p, q, h}=\left(\varphi_{p, q, h}^{j, k}\right)_{1 \leq j, k \leq N_{1}}$, with components $\varphi_{p, q, h}^{j, k}=\frac{2 h}{\pi} \sin (j p h) \sin (k q h)$.

In order to apply theorem 2, we write system (5) as a first order system. Let us then introduce the space $X_{h}=V_{h} \times V_{h}$, which will be endowed with the norm $\left\|\left(\varphi_{h}, \psi_{h}\right)\right\|_{X_{h}}^{2}=\left\|\varphi_{h}\right\|^{2}+\left\|A_{0 h}^{\frac{1}{2}} \psi_{h}\right\|^{2}$. Setting $z_{h}=\left[\begin{array}{c}w_{h} \\ \dot{w}_{h}\end{array}\right]$, equations (5) can be easily written in the equivalent form

$$
\dot{z}_{h}(t)=A_{h} z_{h}(t), \quad z_{h}(0)=z_{0 h},
$$

where $z_{0 h}=\left[\begin{array}{l}w_{0 h} \\ w_{1 h}\end{array}\right]$ and $A_{h} \in \mathcal{L}\left(X_{h}\right)$ is defined by 


$$
A_{h}=\left[\begin{array}{cc}
0 & I \\
-A_{0 h}-h^{2} A_{0 h}-B_{0 h} B_{0 h}^{*}
\end{array}\right] .
$$

It will be useful to introduce the operator $A_{1 h}=\left[\begin{array}{cc}0 & I \\ -A_{0 h} & 0\end{array}\right] \in \mathcal{L}\left(X_{h}\right)$ such that

$$
A_{h}=A_{1 h}-\left[\begin{array}{lc}
0 & 0 \\
0 & h^{2} A_{0 h}+B_{0 h} B_{0 h}^{*}
\end{array}\right] .
$$

We will also need in the sequel the spectral basis of the operator $A_{1 h}$. Moreover, it will be more convenient to number the eigenelements of $A_{1 h}$ using only one index $m$ instead of the couple $(p, q)$. To achieve this, let us first rearrange the sequence of eigenvalues $\lambda_{p, q}=p^{2}+q^{2}, p, q \in \mathbb{N}^{*}$, of the continuous problem in nondecreasing order to obtain a new sequence $\left(\Lambda_{m}\right)_{m \in \mathbb{N}^{*}}$. Then, if

$$
\Lambda_{m}=\lambda_{p, q}=p^{2}+q^{2}, \quad \forall m \in \mathbb{N}^{*}, \forall p, q \in \mathbb{N}^{*},
$$

then we set for all $1 \leq m \leq\left(N_{1}\right)^{2}$, and for all $1 \leq p, q \leq N_{1}$ :

$$
\Lambda_{m, h}=\lambda_{p, q, h}, \quad \varphi_{m, h}=\varphi_{p, q, h} .
$$

Let then $N_{2}(h)=\left(N_{1}\right)^{2}=\left(\frac{\pi}{h}-1\right)^{2}$ be the number of nodes of the finitedifference grid. If we extend the definition of $\Lambda_{m, h}$ and $\varphi_{m, h}$ to the values $m \in\left\{-1, \ldots,-N_{2}(h)\right\}$ by setting

$$
\Lambda_{m, h}=-\Lambda_{-m, h}, \quad \varphi_{m, h}=\varphi_{-m, h},
$$

then it can be easily checked that the eigenvalues of $A_{1 h}$ are $i \Lambda_{m, h}$, where $1 \leq|m| \leq N_{2}(h)$, and that an orthonormal basis of $X_{h}$ formed by eigenvectors of $A_{1 h}$ is given by

$$
\Phi_{m, h}=\frac{1}{\sqrt{2}}\left[\begin{array}{c}
-\frac{i}{\Lambda_{m, h}} \varphi_{m, h} \\
\varphi_{m, h}
\end{array}\right], \quad 1 \leq|m| \leq N_{2}(h),
$$

We are now in position to apply theorem 2 .

\subsection{Checking the assumptions of theorem 2}

To prove condition $i$ ) in theorem 2, we use a contradiction argument. Suppose that there exist $\left[\begin{array}{l}\varphi_{h} \\ \psi_{h}\end{array}\right] \in X_{h}$ and $\omega \in \mathbb{R}$ such that: $A_{h}\left[\begin{array}{l}\varphi_{h} \\ \psi_{h}\end{array}\right]=i \omega\left[\begin{array}{l}\varphi_{h} \\ \psi_{h}\end{array}\right]$. Then, by using the definition (7) of $A_{h}$, we easily obtain that $\psi_{h}=i \omega \varphi_{h}$ and that

$$
\left[\omega^{2}-A_{0 h}-i \omega\left(h^{2} A_{0 h}+B_{0 h} B_{0 h}^{*}\right)\right] \varphi_{h}=0 .
$$

By taking the imaginary part of the inner product of this last relation with $\varphi_{h}$, we get that $\varphi_{h}=0$, and thus $\psi_{h}=0$. Therefore, for all $\omega \in \mathbb{R}, i \omega$ cannot be an eigenvalue of $A_{h}$. Thus, condition $i$ ) in theorem 2 holds true. 
Now, we check condition $i$ ) of theorem 2. Once again, we use a contradiction argument. Let us thus assume the existence for all $n \in \mathbb{N}$ of $h_{n} \in\left(0, h^{*}\right)$, $\omega_{n} \in \mathbb{R}, z_{n}=\left[\begin{array}{l}\phi_{n} \\ \psi_{n}\end{array}\right] \in X_{h_{n}}$ such that

$$
\begin{gathered}
\left\|z_{n}\right\|^{2}=\left\|A_{0 h_{n}}^{\frac{1}{2}} \phi_{n}\right\|^{2}+\left\|\psi_{n}\right\|^{2}=1 \quad \forall n \in \mathbb{N} \\
\left\|i \omega_{n} z_{n}-A_{h_{n}} z_{n}\right\| \rightarrow 0 .
\end{gathered}
$$

To obtain a contradiction, the idea is to decompose $z_{n}$ into a low frequency part and a high frequency part. Then, thanks to the numerical viscosity introduced in the scheme, we prove that the high frequency part tends to 0. Finally, we conclude by using a result on the uniform observability of low frequency packets of eigenvectors.

More precisely, for $0<\varepsilon<1$ and $h \in\left(0, h^{*}\right)$, we define the integer

$$
M(h)=\max \left\{m \in\left\{1, \ldots, N_{2}(h)\right\} \mid h^{2}\left(\Lambda_{m}\right)^{2} \leq \varepsilon\right\},
$$

where the sequence $\left(\Lambda_{m}\right)_{m \in \mathbb{N}^{*}}$ defined in (9) constitutes the sequence of eigenvalues of the continuous problem. The eigenvalues $\Lambda_{m, h}$ for $1 \leq|m| \leq M(h)$ correspond to "low frequencies" and will be damped to zero by the feedback control term $B_{0 h} B_{0 h}^{*} \dot{w}_{h}$. The eigenvalues $\Lambda_{m, h}$ for $|m|>M(h)$ correspond to "high frequencies" and will be damped by the numerical viscosity term. To get the desired contradiction, we follow several steps.

Step 1

$\overline{\text { Let us }}$ prove the two relations

$$
\begin{gathered}
h_{n}^{2}\left\|A_{0 h_{n}}^{\frac{1}{2}} \psi_{n}\right\|^{2}+\left\|B_{0 h_{n}}^{*} \psi_{n}\right\|^{2} \rightarrow 0, \\
\lim _{n \rightarrow \infty}\left\|A_{0 h_{n}}^{\frac{1}{2}} \phi_{n}\right\|^{2}=\lim _{n \rightarrow \infty}\left\|\psi_{n}\right\|^{2}=\frac{1}{2} .
\end{gathered}
$$

Relation (16) follows directly from (14) by taking the inner product in $X_{h_{n}}$ of $i \omega_{n} z_{n}-A_{h_{n}} z_{n}$ by $z_{n}$ and by considering only the real part. By using (14), (16), (8) and the fact that the operators $B_{0 h_{n}}$ are uniformly bounded we obtain that

$$
\left\|i \omega_{n} z_{n}-A_{1 h_{n}} z_{n}+\left[\begin{array}{c}
0 \\
h_{n}^{2} A_{0 h_{n}} \psi_{n}
\end{array}\right]\right\| \rightarrow 0 .
$$

It can be easily that the sequence $\left(\omega_{n}\right)$ is bounded away from zero for $n$ large enough(use a contradiction argument). Therefore, taking the inner product in $X_{h_{n}}$ of (18) by $\frac{1}{\omega_{n}}\left[\begin{array}{c}\phi_{n} \\ -\psi_{n}\end{array}\right]$ and by considering the imaginary part, we obtain that $\lim _{n \rightarrow \infty}\left\|A_{0 h_{n}}^{\frac{1}{2}} \phi_{n}\right\|^{2}-\left\|\psi_{n}\right\|^{2}=0$. This last relation and (13) yield (17). Step 1 is thus complete. 
In order to state the second step, let us introduce the modal decomposition of $z_{n}$ on the spectral basis of $\left(\Phi_{m, h_{n}}\right)_{1 \leq|m| \leq N_{2}\left(h_{n}\right)}$ of $A_{1 h_{n}}$. For all $n \in \mathbb{N}$, there exist complex coefficients $\left(c_{m}^{n}\right)_{1 \leq|m| \leq N_{2}\left(h_{n}\right)}$ such that

$$
z_{n}=\left[\begin{array}{l}
\phi_{n} \\
\psi_{n}
\end{array}\right]=\sum_{1 \leq|m| \leq N_{2}\left(h_{n}\right)} c_{m}^{n} \Phi_{m, h_{n}}
$$

The normalization condition (13) reads then

$$
\sum_{1 \leq|m| \leq N_{2}\left(h_{n}\right)}\left|c_{m}^{n}\right|^{2}=1 .
$$

Step 2

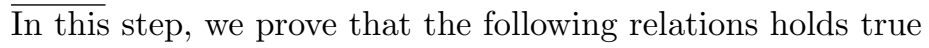

$$
\begin{gathered}
\psi_{n}=\frac{1}{\sqrt{2}} \sum_{m=1}^{N_{2}\left(h_{n}\right)}\left(c_{m}^{n}+c_{-m}^{n}\right) \varphi_{m, h_{n}}, \\
\sum_{M\left(h_{n}\right)<m \leq N_{2}\left(h_{n}\right)}\left|c_{m}^{n}+c_{-m}^{n}\right|^{2} \rightarrow 0, \\
\sum_{1 \leq|m| \leq M\left(h_{n}\right)}\left|\omega_{n}-\Lambda_{m, h_{n}}\right|^{2}\left|c_{m}^{n}\right|^{2} \rightarrow 0 .
\end{gathered}
$$

Note that, roughly speaking, relations (21) and (22) show that the projection of $\psi_{n}$ on the high frequencies tends to 0 as $n$ tends to $+\infty$. Relation (21) follows directly by taking the second component in (19) and by using (12). On the other hand, by using (19) and the fact that $\Phi_{m, h}$ is an eigenvector of $A_{1 h}$ associated to the eigenvalue $i \Lambda_{m, h}$, we have

$$
i \omega_{n} z_{n}-A_{1 h_{n}} z_{n}=\sum_{1 \leq|m| \leq N_{2}\left(h_{n}\right)} i\left(\omega_{n}-\Lambda_{m, h_{n}}\right) c_{m}^{n} \Phi_{m, h_{n}}
$$

From (16) and (21) it follows that

$$
h_{n}^{2}\left\|A_{0}^{\frac{1}{2}} \psi_{n}\right\|^{2}=\sum_{m=1}^{N_{2}\left(h_{n}\right)} h_{n}^{2} \Lambda_{m, h_{n}}^{2}\left|c_{m}^{n}+c_{-m}^{n}\right|^{2} \rightarrow 0 .
$$

Using the expression (6) of $\lambda_{p, q, h}$ and that $\lambda_{p, q}=p^{2}+q^{2}$, it can be easily checked that $\frac{4}{\pi^{2}} \lambda_{p, q} \leq \lambda_{p, q, h} \leq \lambda_{p, q}$, for all $1 \leq p, q \leq N_{1}$, or equivalently

$$
\frac{4}{\pi^{2}} \Lambda_{m} \leq \Lambda_{m, h} \leq \Lambda_{m} \quad \forall 1 \leq m \leq N_{2}(h) .
$$

Relations (25), (26) and (15) imply (22). On the other hand, relations (26) and (25) clearly imply that there exists a constant $C$ independent of $h$ such that 


$$
h_{n}^{4} \sum_{m=1}^{M\left(h_{n}\right)} \Lambda_{m, h_{n}}^{4}\left|c_{m}^{n}+c_{-m}^{n}\right|^{2} \leq C \varepsilon \sum_{m=1}^{M\left(h_{n}\right)} h_{n}^{2} \Lambda_{m, h_{n}}^{2}\left|c_{m}^{n}+c_{-m}^{n}\right|^{2} \rightarrow 0 .
$$

On the other hand, a simple calculation shows that

$$
\left[\begin{array}{c}
0 \\
h_{n}^{2} A_{0 h_{n}} \psi_{n}
\end{array}\right]=\sum_{1 \leq|m| \leq N_{2}\left(h_{n}\right)} \frac{h_{n}^{2}}{2} \Lambda_{m, h_{n}}^{2}\left(c_{m}^{n}+c_{-m}^{n}\right) \Phi_{m, h_{n}},
$$

Relations (27) and (28) imply that

$$
\left[\begin{array}{c}
0 \\
h_{n}^{2} A_{0 h_{n}} \psi_{n}
\end{array}\right]-\sum_{M\left(h_{n}\right)<|m| \leq N_{2}\left(h_{n}\right)} \frac{h_{n}^{2}}{2} \Lambda_{m, h_{n}}^{2}\left(c_{m}^{n}+c_{-m}^{n}\right) \Phi_{m, h_{n}} \rightarrow 0
$$

By using (18), (24) and (29) it follows that

$$
\begin{aligned}
& \sum_{1 \leq|m| \leq N_{2}\left(h_{n}\right)} i\left(\omega_{n}-\Lambda_{m, h_{n}}\right) c_{m}^{n} \Phi_{m, h_{n}} \\
&+\sum_{M\left(h_{n}\right)<|m| \leq N_{2}\left(h_{n}\right)} \frac{h_{n}^{2}}{2} \Lambda_{m, h_{n}}^{2}\left(c_{m}^{n}+c_{-m}^{n}\right) \Phi_{m, h_{n}} \rightarrow 0 .
\end{aligned}
$$

Since the family $\left(\Phi_{m, h_{n}}\right)$ is orthogonal, the above relation implies (23). Step 3

Consider the set

$\mathcal{F}=\left\{n \in \mathbb{N}|\exists m(n) \in \mathbb{Z}, 1 \leq| m(n) \mid \leq M\left(h_{n}\right)\right.$, and $\left.\left|\omega_{n}-\Lambda_{m(n), h_{n}}\right|<\frac{1}{8}\right\}$.

In other words, $\mathcal{F}$ is constituted by those integers $n$ such that $\omega_{n}$ is located in the "low frequency band". We distinguish then two cases:

First Case: The set $\mathcal{F}$ is finite. Then, for the sake of simplicity, we can suppose, without loss of generality, that $\mathcal{F}$ is empty. In this case, all the elements of the sequence $\left(\omega_{n}\right)$ are located in the "high frequency band". By using relation (23) in Step 2 and the above relation, we obtain that < $\sum_{1 \leq|m| \leq M\left(h_{n}\right)}\left|c_{m}^{n}\right|^{2} \rightarrow 0$, i.e. that the low-frequency part of $\psi_{n}$ tends to 0 . Thus, the above relation, (21) and (22) in Step 2 imply that

$$
\psi_{n} \rightarrow 0 \text { in } H
$$

which contradicts (17).

Second case: The set $\mathcal{F}$ is infinite. Then, for the sake of simplicity, we can suppose, without loss of generality, that $\mathcal{F}=\mathbb{N}$. In this case, all the sequence $\omega_{n}$ is located in the "low frequency band". For all $n \in \mathbb{N}$, we introduce the set $\mathcal{F}_{n}=\left\{m \in \mathbb{Z}|1 \leq| m \mid \leq M\left(h_{n}\right)\right.$ and $\left.\left|\omega_{n}-\Lambda_{m, h_{n}}\right|<\frac{1}{8}\right\}$. 
Note that $\mathcal{F}_{n}$ is never empty (since it always contains $m(n)$ ) and represents the collection of low frequency eigenvalues located near $\omega_{n}$. Set then $\widetilde{\psi}_{n}=$ $\frac{1}{\sqrt{2}} \sum_{m \in \mathcal{F}_{n}} c_{m}^{n} \varphi_{m, h_{n}}$. The definition of $\mathcal{F}_{n}$, together with relation (23) of Step 2 imply that

$$
\sum_{m \in\left\{1, \ldots, N_{2}\left(h_{n}\right)\right\} \backslash \mathcal{F}_{n}}\left|c_{m}^{n}\right|^{2} \rightarrow 0 .
$$

Using now relations (21) and (22) of Step 2, we see that (30) exactly states that

$$
\left\|\psi_{n}-\widetilde{\psi}_{n}\right\| \rightarrow 0
$$

The above relation implies that $\left\|B_{0 h_{n}}^{*}\left(\psi_{n}-\widetilde{\psi}_{n}\right)\right\| \rightarrow 0$. This relation together with relation (16) of Step 1 show that

$$
\left\|B_{0 h_{n}}^{*} \widetilde{\psi}_{n}\right\| \rightarrow 0 .
$$

But on the other hand, applying lemma 3.2 in [5] on the uniform observability of low frequency packets of eigenvectors (note that $I_{h_{n}}\left(\omega_{n}\right)=\mathcal{F}_{n}$ ), we get the existence of $\delta>0$ such that for all $n \in \mathbb{N}$, we have

$$
\left\|B_{0 h_{n}}^{*} \widetilde{\psi}_{n}\right\|^{2}>\delta^{2} \sum_{m \in \mathcal{F}_{n}}\left|c_{m}^{n}\right|^{2}
$$

Gathering (30), (32) and (33), we finally obtain that $\widetilde{\psi}_{n} \rightarrow 0$ in $H$. By using (31), we obtain that $\psi_{n} \rightarrow 0$ which contradicts (17). The proof of theorem 1 is now complete.

\section{References}

1. Glowinski, R., Li, C.H, Lions, J-L. : A numerical approach to the exact boundary controllability of the wave equation. I. Dirichlet controls: description of the numerical methods. Japan J. Appl. Math., 7, 1-76 (1990)

2. Infante, J. A., Zuazua, E. : Boundary observability for the space semidiscretizations of the 1-D wave equation. M2AN Math. Model. Numer. Anal., 33, 2, 407-438 (1999)

3. Jaffard, S. : Contrôle interne exact des vibrations d'une plaque rectangulaire. Port. Math., 47, 4, 423-429 (1990)

4. Liu, Z., Zheng, S.: Semigroups associated with dissipative systems, Chapman \& Hall/CRC, Boca Raton (1999)

5. Ramdani, K., Takahashi, T., Tucsnak, M. : Internal stabilization of the plate equation in a square : the continuous and the semi-discretized problems. J. Math. Pures Appl., To appear

6. Tcheugoué Tébou, L. R., Zuazua, E. : Uniform exponential long time decay for the space semi-discretization of a locally damped wave equation via an artificial numerical viscosity. Numer. Math., 95, 3, 563-598 (2003)

7. Zuazua, E. : Propagation, observation and control of waves approximated by finite difference methods. SIAM. Rev., 47, 197-243 (2005) 